\title{
USING AGENT MODELS AND DATA FARMING TO EXPLORE NETWORK CENTRIC OPERATIONS
}

\author{
Henrik Friman \\ Swedish National Defense College \\ 117 Vahalavagen \\ Stockholm, SWEDEN
}

\author{
Gary E. Horne \\ Referentia Systems Incorporated \\ 550 Paiea St. \\ Honolulu, HI 96819, U.S.A.
}

\begin{abstract}
Network Centric Operations are difficult to quantify in many respects with models or other methods. Data Farming is a methodology and capability that makes use of high performance computing to run models many times. In the case of agent-based models that are relatively small, many runs can be performed in a short period of time. This capability gives modelers and their clients the enhanced ability to discover trends and outliers in results in a variety of areas. In this paper the authors discuss some notional efforts to begin to explore questions in the area of network centric operations using the agent model MANA and Data Farming. By observing the network behaviors and the output for traditional and Information Age warfare, we have created comparisons that illustrate when networked forces outfight non-networked forces
\end{abstract}

\section{BACKGROUND}

Most western armed forces today are in what could be described as a transformation mode that has its origins in the information society's new technologies in combinations with new threats. To support the military force's transformation, new concepts such as Network Centric Operations and Distributed Operations have been developed (US Office of Transformation 2004). A key assumption behind the transformation is that information dominance and information superiority increase the probability of success in combat situations. But what does this mean in practical terms? Researchers have attempted to investigate different information perspectives relating to effects or system outcomes, but few specific or practical results have been reported. One example is Leavitt (1978), who showed in simple structures of communication networks in a group that individuals seem to set limits on the group's performance. Groups whose problems require the collation of information from all members most likely work faster when one position is highly centralized and the others are relatively peripheral. On the other hand, morale and creativity might be better when the communication network is more egalitarian and when each person has more than one source of information. Highly centralized groups may often be useful for their consistency with general organizational designs, their speed, and their controllability; but they can also be used to protect a superior's weaknesses from being exposed to subordinates, and vice versa (Leavitt 1978, page 243). So, there is literature addressing problems from areas other than the military which could be used as references and inspiration for investigations within the military field.

Vice Admiral Cebrowski addressed this issue by stating his hypothesis that: Networked forces outfight nonnetworked forces (US Office of Transformation 2004). This hypothesis could be considered the "holy grail" for the war fighters within the Information Age. This paper contains illustrative explorations that test Cebrowski's hypothesis by using Agent Based Models and Data Farming. The attempt is to clarify some of the basic assumptions behind the transformation and to illustrate what the results might mean to further development. This work is based on literature and results from a relatively limited number of runs with the agent based model MANA. This work simply gives indications of trends and structures rather than definitive answers.

\section{WHAT DO WE MEAN BY OUTPUT?}

First, we need to consider what we mean by output. (There are a great number of terms to describe the output from the military system, such as mission effectiveness, success, performance. In order to not get caught in an argument about definitions of terms, we in this text will use the term output, without doing any further specification, and by that cover all conceivable outputs.) One of the leading establishments of force transformation is US Joint Forces Command (JFCOM). JFCOM has defined the desirable output in terms of Effects Based Operations (EBO). EBO is "a process for obtaining a desired strategic outcome or 'effect' on the enemy, through the synergistic, multiplica- 
tive, and cumulative application of the full range of military and nonmilitary capabilities at the tactical, operational, and strategic levels" (US Joint Forces Command 2005). Central to this definition is the term effect which has been defined as "the physical, functional, or psychological outcome, event, or consequence that results from specific military or non-military actions" (US Joint Forces Command 2005). Effect is not only the hard numbers that are created by military actions; it is also considered to be outcomes such as will to fight. In this sense, EBO could be considered parallel to Sweden's concept of maneuver thinking, which is described as physical, conceptual and emotional factors (Swedish Military Doctrine 2002). The definitions above lead us to the argument that output in the Information Age is a result of network behaviors.

It is also important to point out that EBO addresses all levels of war, and promotes synergistic, multiplicative, and cumulative applications. Or, as Deptula (2001) stated "the important measure is not the targets destroyed but rather the effect on the enemy's capabilities and actions." In this study we are more focused on concrete tactical examples rather than trying to illustrate forces in the information age with more abstract strategic and operational examples.

Output in a general sense could be formulated in terms of optimizations of performance or balancing of performance. Output in technical systems is often described as optimizations of performance, which is a trade-off between quality, quantity and cost aspects. Through operational research we have learned to optimize processes based on quantitative data and statistical analyses; the baseline for achieving such data is with systems that are well defined, and which components (dots - in agent based models the components are represented as dots) have recurrent functions (e.g., Alberts and Hayes 2003).

Output of human systems in military contexts are more often described in terms of survival, which concerns what could be called balancing of performance rather than optimization of performance. The dots and interrelations between individuals within and between groups are normally described in terms of qualitative data and analyses. Outputs from measurements of human systems at group levels can, under certain circumstances, be generalized and treated by statistics in order to find general human patterns. Studies of individuals are normally treated as specific case studies and the results are unique to the situation but are difficult to convert to other situations. VADM Cebrowski addressed this issue as the need for a well balanced force.

The use of optimizing measurements on human systems will, in most cases, fail since survival is of higher value then just finding the highest optimized effects. Individuals and groups could show willingness to self sacrifice in order to create survival for their families, but will not do

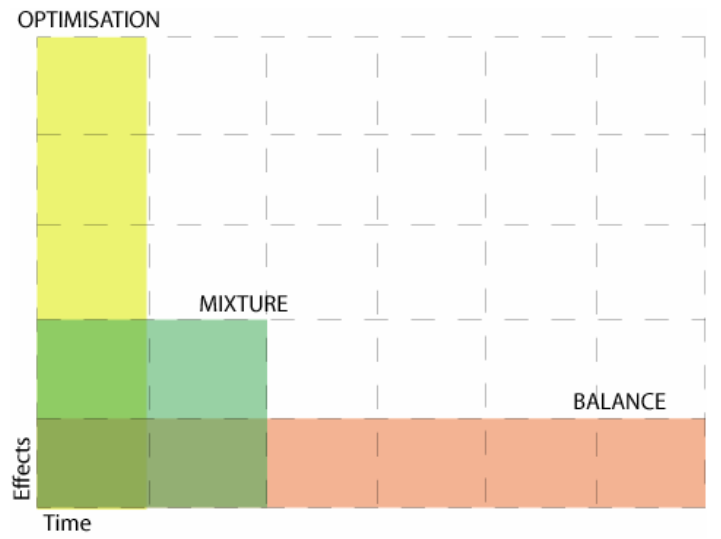

Figure 1: Outputs of Optimization and Balance

that just to be "cost" effective. It is hazardous to argue balanced solutions based on optimized measurements. Optimization does not reflect a balanced approach to military outputs as it does not include such intangible factors as human emotions feelings and minds, such as fear and morale, which are central for humans and are not possible to optimize. Depending upon whether we define the problem in terms of an optimization issue rather than a balancing issue, we can employ a different approach and find different results as output.

\section{AN ILLUSTRATIVE EXAMPLE OF NETWORK BEHAVIORS}

By observing the network behaviors and the output within the traditional and Information Age warfare, in this section we describe a comparative study that illustrates when networked forces outfight non-networked forces.

\subsection{Assumptions Behind the Study Design}

In order to illustrate the information impact on network behaviors, two key dots are assumed to be essential to the arguments about Distributed Operation concepts, information superiority and ways of structure (network topologies.) In its most general original form of information superiority, it is assumed that the part that has the most information also has the highest probability to win the fight (e.g., Alberts and Hayes 2003). The second issue is the organizational structure of information nodes which are assumed to be the hierarchy of traditional warfare and the web (network) of Information Age warfare.

Traditional warfare structure is often described using a hierarchy that is controlled by the top and fed down from the bureaucracy (Alberts and Hayes 2003). Information Age structures are often argued to be web structures where 


\section{Friman and Horne}

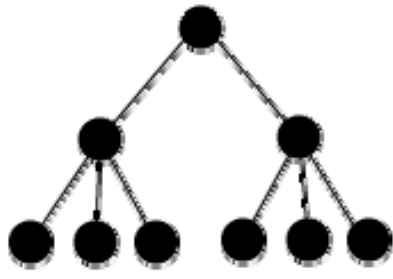

Hierarchy

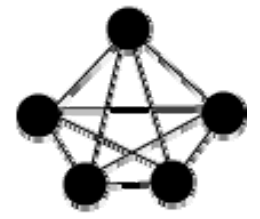

WEB
Figure 2: Network Topologies

the components are connected to each other (Leavitt 1978). It is important to note that there are several types of networks (such as organizational, social, and technical) which all have differences. To illustrate the relationship between information and behavior, we will control the communication between the active components within the systems, and observe the differences in behavior and output.

The comparative study will be done with four scenarios in which the Data Farming will take place on different information settings within the two different structures (hierarchy and web). The design is based on the assumptions that qualities within communication are directly related to information qualities and also directly influence improvement of decision making. This assumption could be debated, but will be used in this early stage in the illustrative example.

\subsection{Agent Models and Data Farming}

We choose to use Agent Based Models since the number of qualities on the information variables was assumed to be relatively limited and the main interest of this study was to discover network behaviors. An Agent Based Model could generate simple scenarios in which key relations between dots are described. Each component in the model could be given different settings (sensor, effecting, and hovering attributes) that represent the information qualities such as latency, communication range, communication capacity, links, information age, sensor range, broadcast range, different leadership styles, and obedience to orders or tactics. Agent Based Models give us the ability to quickly run multiple scenarios as well as scenario variants that represent the area of interest (Horne 2001, Horne and Meyer 2004).

By running the model multiple times in a supercomputing environment, we see that the results from the Agent Based Model could provide insights on Coevolving Landscapes of the dots behaviors. Data Farming involves the investigation of a wide number of variables across a wide range of values. In essence, the user is attempting to model many combinations and variations within the data space and "grow" data in an iterative process attempting to answer questions at hand. Data Farming is applied to distillations, models that can be used to abstract the essence of a situation. It is important to note that the results of Data Farming are not just statistics coming out of multiple runs of a model but also give qualitative data sets of insights documented in research notes. The results are also notes taken at the discussions where the model is set up, watching the behaviors within the model when it runs, and putting notes and results together. Data Farming, of course, could be performed using other models than agent based.

\subsection{Setting Up the Study}

The set up for the study is based on the NATO-sponsored group SAS-050, which is exploring new C2 concepts and capabilities formulation of network centric operations. The problem formulation is based on five controllables which are translated into the variables within the agent based model that describes the attributes of the dots. The controllables are described within the model in Table 1.

Table 1: Description of Controllables in the Model

\begin{tabular}{ll}
\hline Controllable & Description in model \\
\hline Quality information & $\begin{array}{l}\text { sensors and communica- } \\
\text { tion }\end{array}$ \\
Share information & communication \\
Collaboration mechanism & communication \\
Shared ability & formation and success \\
Network force & behavior \\
\hline
\end{tabular}

Here we use the agent model MANA developed in New Zealand and part of the suite of models in the Project Albert Data Farming Environment at the Maui High Performance Computing Center. Situation awareness and situation understanding could not be directly described in MANA because there are cognitive issues related to the users rather than to the variables within the system set-up. Nor could decision making qualities be measured objectively. So, the hypothesis chain is simplified to information access which will influence the agents' behaviors that improve the ability to synergize actions. In this study we have been skipping the middle level in problem formulation and directly connecting assumption inputs. That could be described with comparative analyses of differences in objective functions of the model variables.

In order to make a comparative measurement, we used agility. Distributed operation agility is considered to be a key factor for success (US Office of Transformation 2004). In MANA we used force to describe the effecting attributes. Variables used for effecting attributes will generate causalities based on the agent's information about the opposite force. Secondly, $C 2$ is understood as sensors. Sensors will generate information about their own and opposite forces. Thirdly, information's accessibility and quality are the sources that make success. In this study information is described with communications variables which are the control variables (Data Farming parameters). Agility 
should be understood as freedom of action (ability to maneuver), and is described within the model as how the dots are moving which illustrates the dots' behavior.

The measurement of outputs is based on balance strategy rather then on optimization strategy. This means that reaching the goal with as low casualties as possible is preferable to reaching the goal within as few time steps as possible. Measurements of effects are done by a comparison between blue and red casualties, and success is when blue reaches the goal with as low casualties on both sides as possible. The Data Farming parameters are given in Table 2.

Table 2: Data Farming Parameters

Parameters

Blue force

\begin{tabular}{ll}
\hline Communication range & $50-200$, steps $=50$ \\
Communication capacity & $20-100$, steps $=20$ \\
Communication Latency & $0-15$, steps $=5$ \\
Communication Accuracy & $25-100 \%$, steps $=25$ \\
Communication reliability & $25-100 \%$, steps $=25$ \\
Inorganic vs Organic Info & On $/$ Off \\
\hline
\end{tabular}

\subsection{Results and Findings from the Data Farming}

As shown in Figure 3, this scenario has a blue force with the task to reach the blue flag, and a red force that is divided into three units and the formation of a trap, "killing sack."

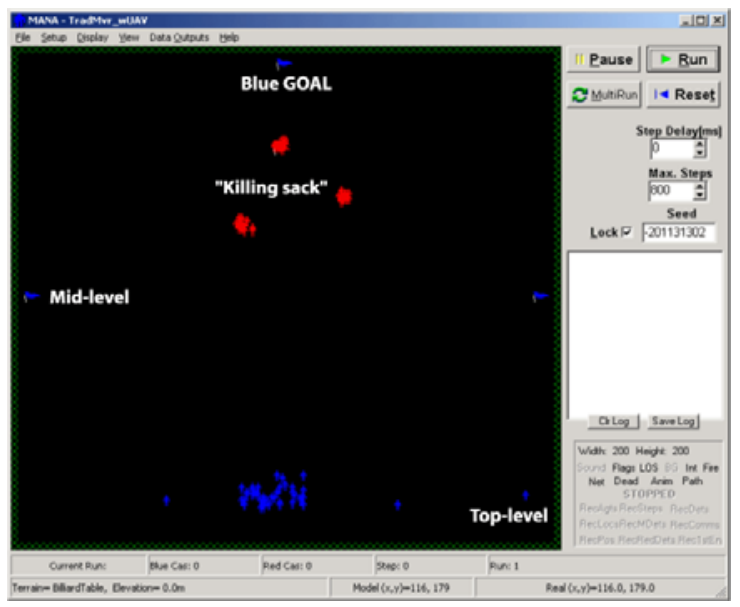

Figure 3: Scenario Setup in MANA

\subsubsection{Scenario A - Base Case}

Settings. As shown in Figure 4, blue forces move onto the blue target with its own sensors. Communication is set to share the information between all the dots (Traditional warfare case). In this scenario both the middle-level and the top-level are inactive.

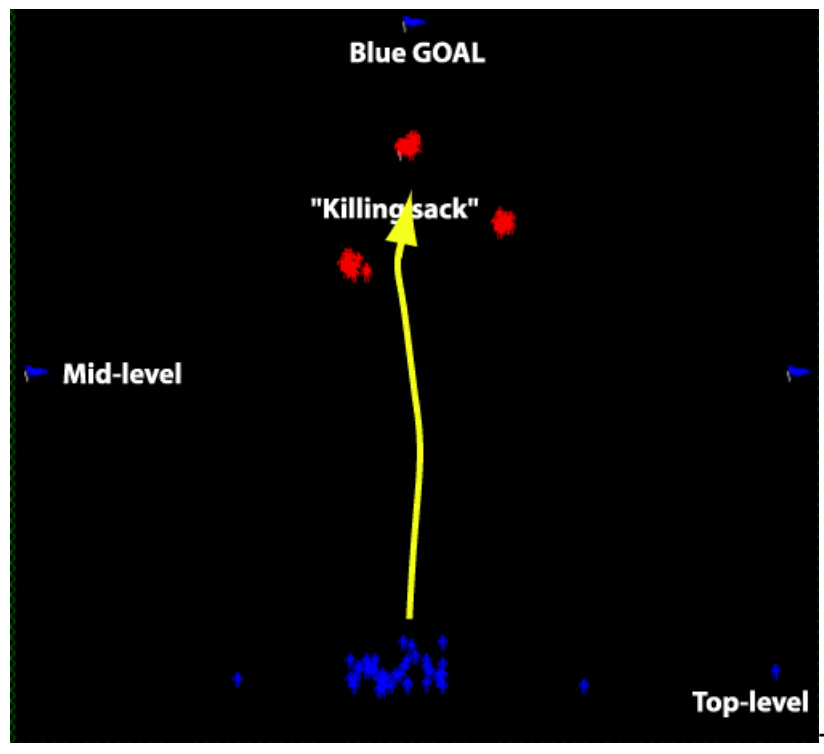

Figure 4: Blue Force Trapped in the "Killing Sack"

Results: As depicted in Figure 5, only in limited runs (2 out of 30) will the blue force reach its goal in this scenario. Most time the time blue force will be trapped in the "killing sack."

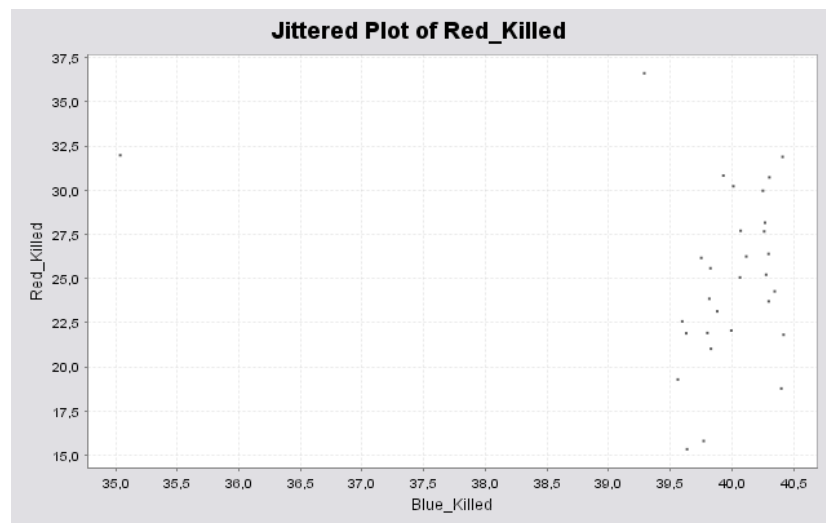

Figure 5: Jittered Plot for Scenario A

In Figure 5 we can see that blue had sustained heavy losses (casualties) compared with the losses sustained by red force. This example follows historical based studies that argue that attacking force needs to outnumber defending forces. We have not investigated which relative numbers of force components are needed to get an equal winning situation between blue and red since it was not the aim of this study.

\subsubsection{Scenario B - Base Case with Information Superiority}

Settings. As shown in Figure 6, Scenario B is based on scenario A, but now blue force is given information superiority and can sense everything in the battle space. 


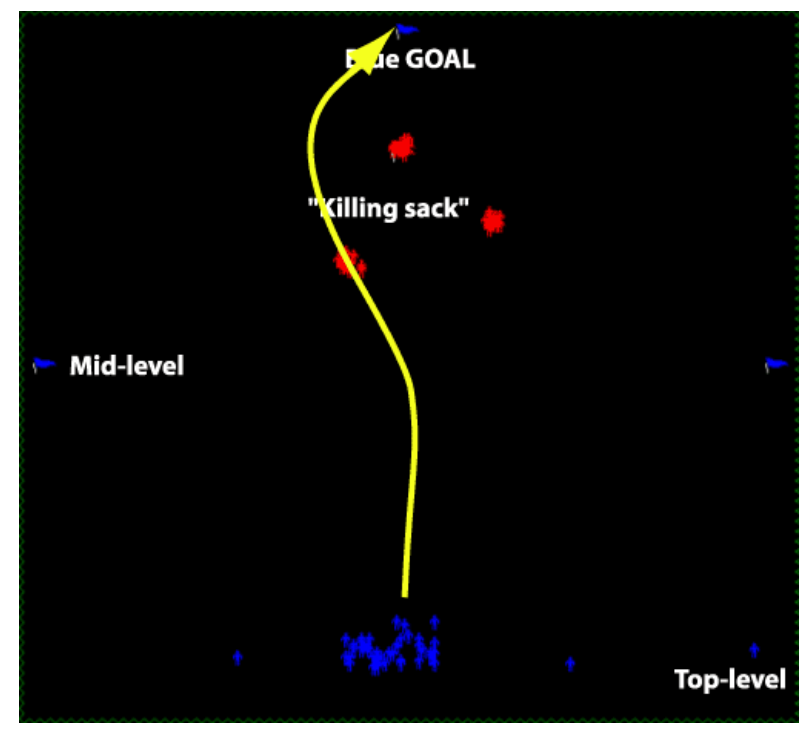

Figure 6: Blue Force Start to Maneuver to Reach the Goal

Results. As displayed in Figure 7, blue force is now reaching the goal in most cases. Instead of getting captured within the trap of the blue force it picks a fight with a smaller part of the red force and maneuvers around the rest to reach the goal.

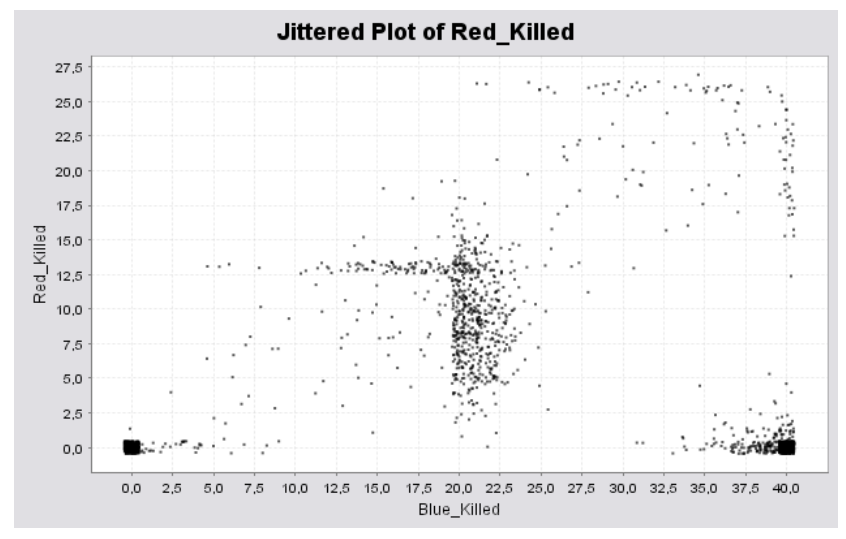

Figure 7: Jittered Plot for Scenario B

Figure 7 shows a much higher probability for the Blue force to reach the goal, but still with high casualties.

\subsubsection{Scenario C - Hierarchy Structure}

Setting. As shown in Figure 8, blue force information is distributed from top to bottom through a hierarchy structure. Here three types of blue dots were introduced. Low level dots actually move to the goal, and have the attributes to cause effects and take casualties. The mid-level dots can sense part of the battle space and pass on the information to lower levels. The mid-level also has the capability to pass on information from the top level. The top level has better sensors than the mid-level. This setup is an attempt to describe a top feeding hierarchal structure.

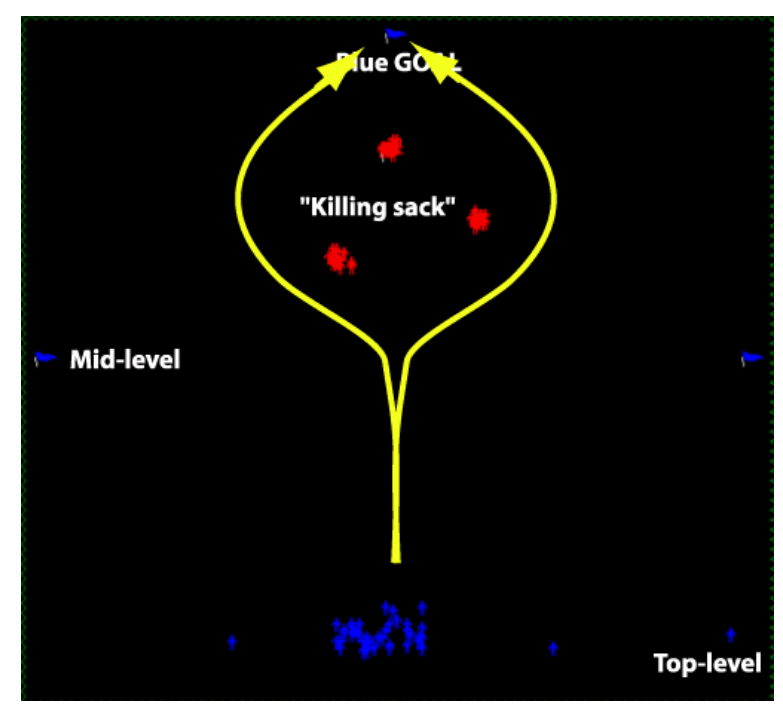

Figure 8: Blue Force Makes Double Envelopment Operation

Results. As depicted in Figure 9, blue force is reaching the goal in most cases by making a double envelopment operation.

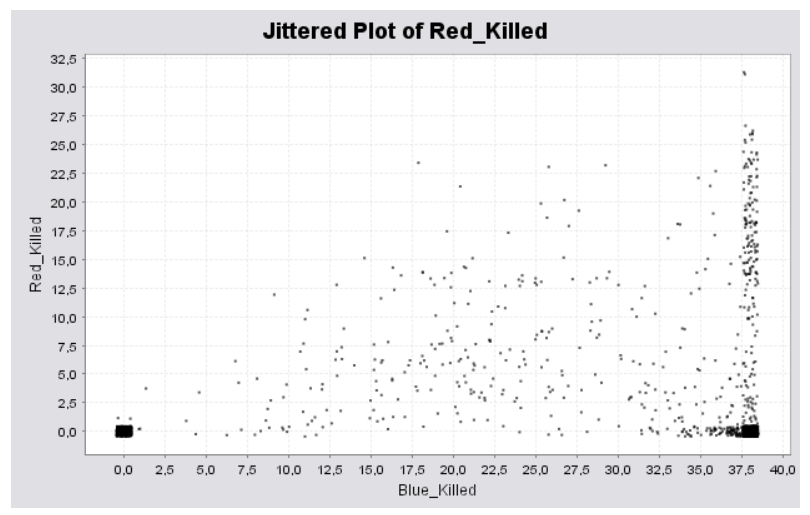

Figure 9: Jittered Plot for Scenario C

Figure 9 shows a less random pattern of outputs. The goal could be reached without actually outfighting all the opposite forces, but rather by moving around. This strategy results in a higher number of casualties on the Blue side which might be due to the fact that the Blue force is attacking in this scenario.

\subsubsection{Scenario D - Hierarchy Structure with Information Superiority}

Settings: As shown in Figure 10, Scenario D is based on Scenario $\mathrm{C}$ but now the blue force is given information $\mathrm{su}$ periority and can sense everything in the battle space. 


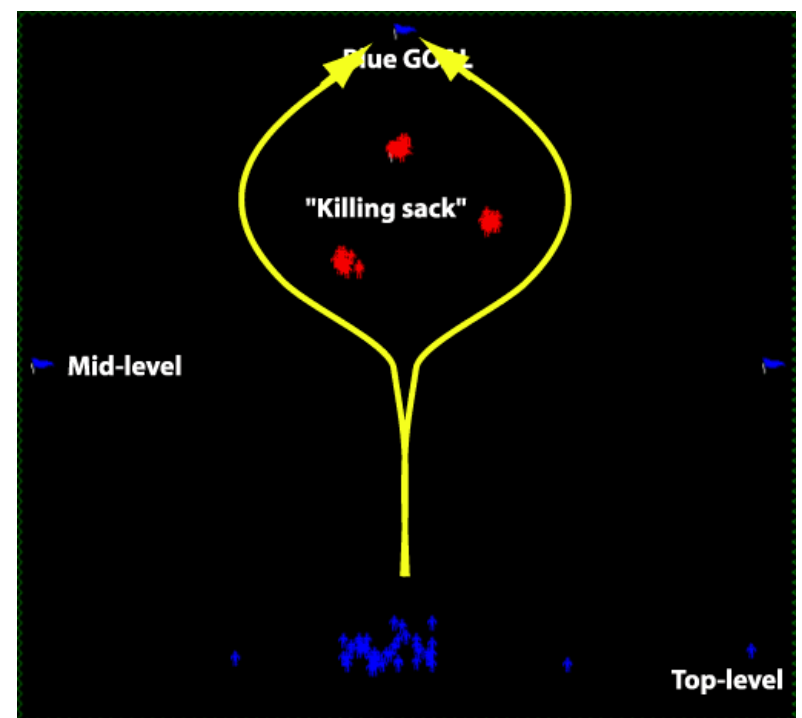

Figure 10: Blue Force Makes Wider Double Envelopment

Results. Figure 11 shows that the blue force is reaching the goal by making a tighter double envelopment operation.

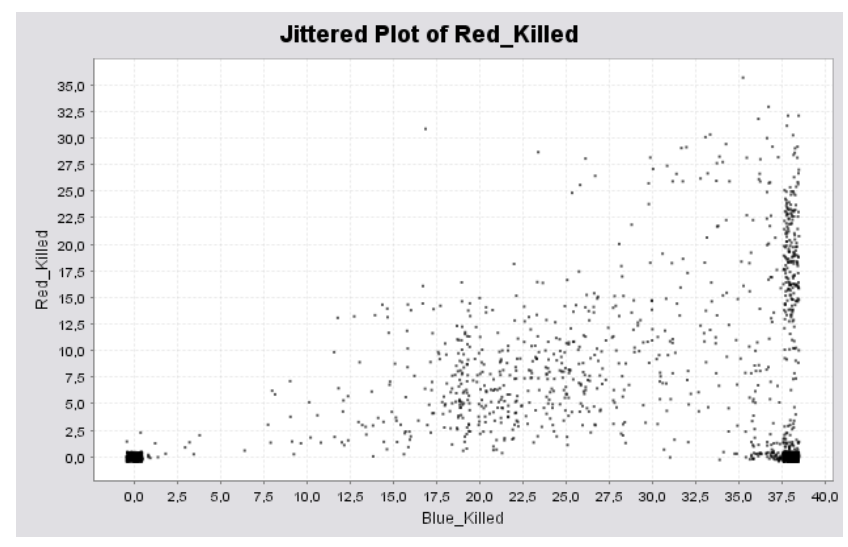

Figure 11: Jittered Plot for Scenario D

The tighter maneuver requires fewer time steps for blue to reach the goal. In Figure 11 the casualties now decrease for the Blue forces, but still attacking a defending force is shown to be a high risk operation.

\subsection{Results}

In this study we aimed to create illustrative examples that tested VADM Cebrowski's hypothesis, that networked forces outfight non-networked forces. By using the SAS050 problem formulation of Information Age warfare, by using the Agent Based Model MANA, and by using Data Farming we found support for the hypothesis.

\subsection{Important Notes from the Discussions Setting up the Models}

Through the whole study the researchers kept a notebook for insights and reflections. Some of those notes, we think, are of such importance that they should be shared in relation to the achieved study above.

Size. In this study we have used an equal size on each side. It is reasonable to believe that alterations in size between the two forces will make differences. But, in this scenario we used a balance strategy, and tried to reach the goal with as little casualties as possible rather than outfighting the enemy. So, in these scenarios where the blue forces actually maneuver around the red forces, this alteration might have limited effects. The question of size within the Information Age environment needs to be investigated further.

Number of components. In traditional warfare with force-on-force situations the quality and the number of resources on the battlefield are the main focus for effects evaluations. The effect of the quality is improved by developing of multifunctional platforms. The question about the number of components could not be answered without first stating the functionality and type of component we assume to be in the future. The first assumption we should try to investigate are the differences between multifunctional and more simplified platforms in which we split sensors, weapons, and $\mathrm{C} 2$ cells into separate components.

Importance of Formation. Setting up the scenario with the formation of dots at the battle space made a direct impact on how they later behaved through the runs. Even though the military forces transform into distributed forces or self-synchronized units, the formation between objects will matter! The ability to make Synergistic Actions is based on positions and quality of information, which needs to be further explored.

Reserve. The question is: If we have networked forces, do we really need a force reserve in the traditional sense? Distributed Networked forces could communicate in near real time and in longer terms, if they used a component that couldn't normally be used. The reason for having a reserve is to retain freedom of action and, with that, handle uncertainty. Freedom of action with Distributed Networked forces could be retained by improving communication and the radius of action for long distance precision weapon. So, reserve in Information Age environment will perhaps then mean more robustness in sensors, and reliability to access nets and weapons. 


\section{DISCUSSION}

The first natural question is: what is new? The impression is that the model describes traditional force on force situations. Model settings sometimes need to be set as stereotypes to make the illustrative case of diversities between different settings. The network behaviors have special interests in agent based settings that different communications and information variables give to certain situations. It is a risk that the used models investigate general net problems rather than address the specific Information Age problems. We have attempted to state the problem in relevant terms so it makes sense to run the models at a tactical level and that the results of interest to transformation development could be investigated.

The agent based approach makes it possible to run many scenarios within different settings in order to explore beconcepts and thought described in literature about transformation. Cognitive factors such as situational awareness and understanding could not be properly described in models since they are individually related and hard to quantify. But with the assumption that such factors influence different information qualities, part of the problem could be described within the model.

This work shows that Agent Based Models and Data Farming can be used to explore Information Age questions and the Network Centric Operational Approach. The agents clearly show different network behaviors depending upon the different settings that were used. And the outcome of adding an all-knowing view to a traditional information structure had limited effects on the results, which leads us to believe that technological development and organizational changes are necessary to take advantage of the effects of new technologies such as a sensors, unmanned vertical assets, etc.

\section{ACKNOWLEDGMENTS AND NOTES}

The authors would like to acknowledge all of the Project Albert collaborators especially Sarah Johnson and Danielle Martin for their insight, time, and assistance. This paper contains facts and opinions which the authors alone considered appropriate and correct for the subject. It does not necessarily reflect the policy or the opinion of any other person or agency.

\section{REFERENCES}

Alberts, D. and Hayes, R. 2003. Power to the Edge. Command and Control Research Program., Washington, D.C.

Deptula, D. A. 2001 Firing for effects. AIR FORCE Magazine 84 (4): 46-53. Available online via www.afa.org/magazine/Apri12001/0401e ffects.pdf [accessed September 16, 2005].
Horne, G. 2001. Beyond Point Estimates: Operational Synthesis and Data Farming. Maneuver Warfare Science 2001. United States Marine Corps Project Albert. Quantico, Virginia.

Horne, G. and Meyer, T. 2004. Data Farming: Discovering Surprise. Proceedings of the 2004 Winter Simulation Conference. Denver, Colorado.

Leavitt Harold. J. 1978. Managerial psychology (4ed), Chicago: The University of Chicago Press.

Swedish Military Doctrine. 2002. Developing a Swedish military doctrine - a documentation of the doctrine process. Available online via www.kkrva.se/Artiklar/011/011e/ahlgr en.html [accessed September 16, 2005].

US Joint Forces Command. 2005. Joint Forces Command Glossary. Available online via http: / /www.jfcom.mil/about/glossary . htm [accessed September 16, 2005].

US Office of Force Transformation. 2004. Transformation trends, report 7 December 2004. Available online via http://www.oft.osd.mil/ [accessed September 16, 2005].

\section{AUTHOR BIOGRAPHIES}

HENRIK FRIMAN, Ph.D. is Associated Professor at the Swedish National Defence College (SNDC), Stockholm, Sweden. The SNDC specializes in command and control research of military science. His professional background is in strategic and innovation management, time research, futurology, and command and control systems. His email address is henrik. frimaneswipnet.se.

GARY E. HORNE is the founding member of the Data Farming Team at Referentia Systems Inc. He holds a B.S in Mathematics and an M.Ed. in Education from the University of Maryland and a D.Sc. in Operations Research from the George Washington University. He is the originator of the Data Farming methodology and the Director of Project Albert. His email address is ghorne@referentia.com. 